\title{
Representation of Multi Resolution Image using Multi Wavelet Transform with Combined 2-D and 1-D Directional Filter Banks
}

\author{
M.Kamaraju, Ph.D \\ Department of ECE, Gudlavalleru \\ Engineering College, Gudlavalleru, \\ India
}

\author{
T.Krishna Sagar \\ PG Student Gudlavalleru \\ Engineering College, \\ Gudlavalleru, India
}

\author{
K.Ramanjanyelu, Ph.D \\ Department of ECE, \\ PVPSiddharthaEngineeringCollege \\ Vijayawada, India
}

\begin{abstract}
Multiresolution analysis is a design method for most of the practically relavant discrete wavelet transform. Multiresolution approaches provide a powerful tool for image processing. Combined 2-D and 1-D directional filter banks are used for the visual quality improvement and have higher PSNR than the predictable separable wavelet transform. Multiresolution image that makes image look good on all screen resolution. Multiresoution keeps each screen pixel filled with at least one image pixel, upto maximum resolution of the image. Here in addition being proposed multi wavelet transform that exploits the multiresolution image and can efficiently represent the sharp edges of the image. The development in this paper draws the results regarding the multi wavelets appear to excel at preserving high frequency content. In particular, multi wavelets better capture the sharp edges and geometric patterns that occur in images.
\end{abstract}

\section{Keywords}

Directional filter banks, 2-D filter banks, multi wavelet transform.

\section{INTRODUCTION}

Digital image processing is defined as analyzing and manipulating images. The traditional scheme to grasp multiresolution image representation (MIR) is to apply 1-D filters separately in horizontal and vertical directions, commonly referred to as "separable" transform. in contrast, "nonseparable" transforms consist of 2-D filters and 2-D downsampling matrices which cannot be factorized into 1-D filter/downsampling pairs.
The contourlet transform is one of the most well-known transforms in this category, which obtains MIR as laplacian pyramid. It is strongly related to curvelets and it succeeded to represent images by using fixed (nonadaptive) implementation, which is suitable for simple representation of images. However, it requires several sets of 2-D FBs which have specific pass band supports. It shows improvements on image de-noising; however, it still has redundancy and does not use the traditional wavelet transform filters. This implies that we cannot apply it as a simple alternative of the wavelet transform.

Furthermore, a number of transforms classify and decompose images into blocks with similar directional features and process each decomposed block depending on directional information.

By transforming along the curves in images, the directional wavelet transforms preserve both high-frequency and low-frequency information even in low bit rates where the separable wavelet transforms often yield blurred artifacts. However, they have some drawbacks compared to the multiwavelet transform. The main negative aspect is the computation cost to determine the curves in images. At each pixel, transforms are needed for several directions to decide the direction which maximizes the energy in LL sub band (i.e., minimizes the energies in LH, HL, and HH sub bands). Furthermore, they need to transmit the side information of the curves to decoder. Although this barely affects the entire bit budget to be encoded, it usually requires a careful manipulation to store or transmit since it should be lossless data. In this paper, two TODFBs are provided to use the effective existing framework of the separable wavelet transform. Their frameworks are shown in Fig1.

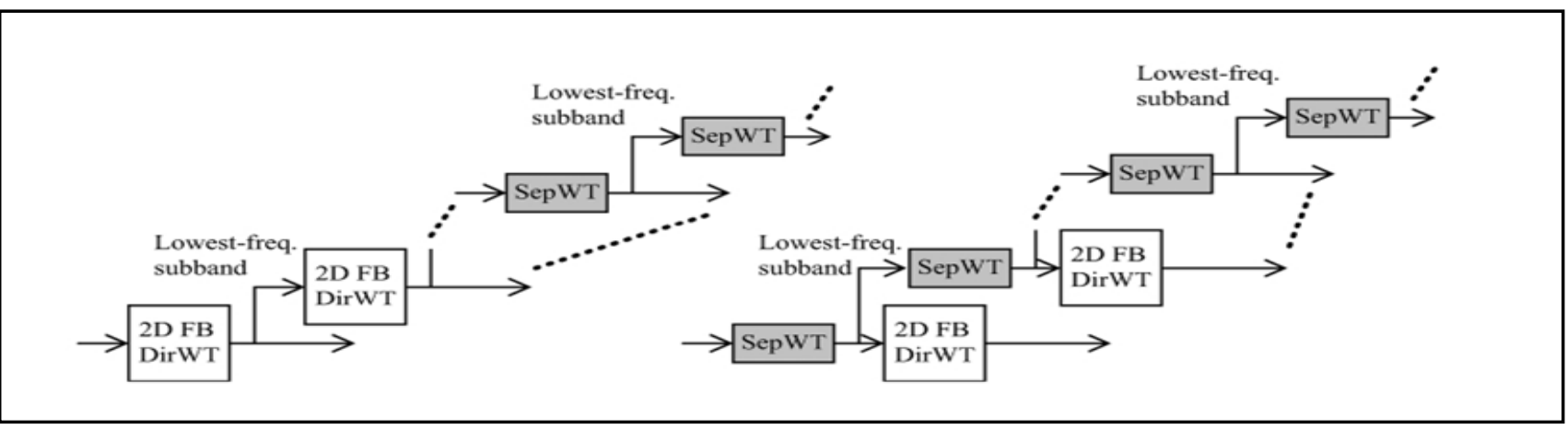

Fig1. Two TODFB Frameworks Where Sep-wt and Dir-wt Transforms Referred to Separable Wavelet Transform and Directional Wavelet Transform.

Combination is used to decompose high-frequency sub bands obtained from the separable wavelet transform in the other TODFB (right side of Fig1.).It is worth noting that these TODFBs use different 2-D FBs but have the same purpose the first stage in the TODFB.Extract the curves in the sub bands by the 2-D FBs, and the second stage reduce the redundancy in these curves by the directional wavelet transforms. They do not require any adaptive processing as in the conventional directional wavelet transforms.
This paper is organized as follows. Section ii gives reviews of the traditional 2-D FB-based MIRs and the multi directional wavelet transforms. The first TODFB shown in Fig1(a) is presented in section iii. In section iv, the second, i.e., the structure of Fig1 (b), is presented. The proposed structure and their comparison with those of the traditional MIRs are shown in section iv, and vi represents the results. Finally, section viii concludes the paper. 


\section{LITERATURE REVIEW}

\subsection{Directional Filter Banks and contourlets}

The directional FB was first proposed by Bamberger and smith, and then their improved tree-structure version. The key idea is to divide the frequency plane into several parts which correspond to the specific frequency directions. The frequency plane partition of a directional FB with eight sub bands is represented in Fig2. With the improved structure, they are realized by using quincunx FBs.

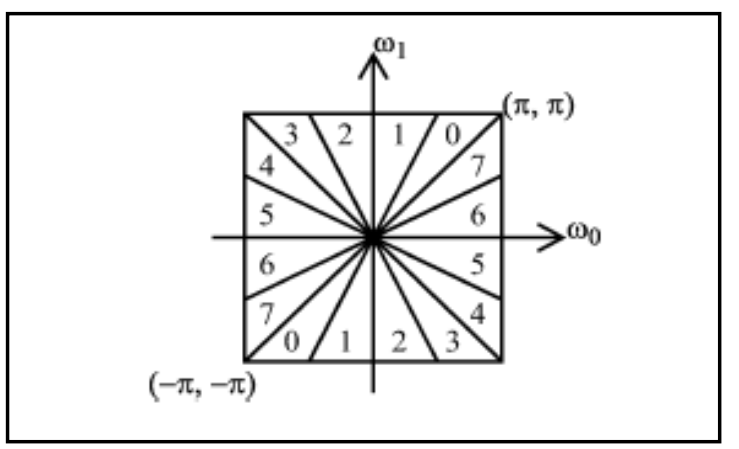

Fig2. Frequency Partition for the Directional FB with

\section{Eight Sub bands.}

The decomposition level can divide orientations in images effectively, although all the sub bands keep low-frequency energy. It often becomes a dis-advantage in practical image processing such as compression, since images usually have large amounts of DC energy. In other words, to concentrate DC energy into the lowest sub band is an important issue for such applications.

The contourlet transform can be realized by applying the directional FB to the highpass sub band which is obtained from laplacian pyramid. It solves the problem of the directional FBs as mentioned previously. However, it is a redundant transform, i.e., it is regarded as a type of overcomplete transforms. Indeed, some image coding applications using the contourlet with its parent-children relationship. Unfortunately, they are inferior to the traditional wavelet transform image coder especially for natural images.

\subsection{Multi Directional Wavelet Transforms}

The standard 2-D wavelet transform is widely used in image processing with a great success. It is a separable transform allowing for a simple filter design and low computational complexity. However, it fails to capture efficiently phenomena in images in directions other than the horizontal and vertical. Recently, many approaches have been made to exploit geometrical features in images. These include curve lets, contour lets, wedge lets, directional filter banks, etc. Although they provide sparser representations in the transform domain, they are computationally complex or involve complicated filter design. The goal is to retain computational simplicity of the standard wavelet transform and to make use of directional coherences in images. We propose multi-directional wavelet transform that is capable of providing efficient representation of images. The proposed transform can be easily applied in all areas of image processing where the standard 2-D wavelet transform is used.

\section{2-D, 1-D DIRECTIONAL FILTER BANK (TODFB)}

\subsection{TODFB with Quincunx Filter Bank}

This section introduces the TODFB using quincunx FB as its 2-D stage. The image is primarily decomposed by the quincunx FB, and then its high-frequency sub bands are transformed by the directional wavelet transform for specific directions. The entire decomposition is shown in Fig3. In this Fig, arrowheads in "directional wavelet transform stage" represent the transform directions. For sub bands 1, 2, and 3, the 9/7 directional lifting wavelet transform is applied in the direction $(0,1),(-1,1)$, and $(1$, $1)$ first, and then in the direction $(1,0),(1,1)$, and $(-1,1)$, respectively. The downsampling matrix for the low-frequency resolution (sub band 0 ) is always $Q^{2}=\operatorname{diag}(2,2)$, which is the same as that of the separable wavelet transform. Consequently, the low-resolution image is transformed recursively by the TODFB or the separable wavelet transform. Here after, this kind of TODFBs is called Q-TODFB where "Q" stands on the initial letter of Quincunx FB.

\subsection{TODFB with Diagonally quadrant Filter Bank}

In this subsection, another type of TODFB is presented. It uses the separable wavelet transform as a preprocessing in each level, and then the 2-D FBs and directional wavelet transforms further decompose the high frequency directions. We describe the details of this TODFB called D-TODFB where "D" stands on the initial letter of diagonally quadrant FB.

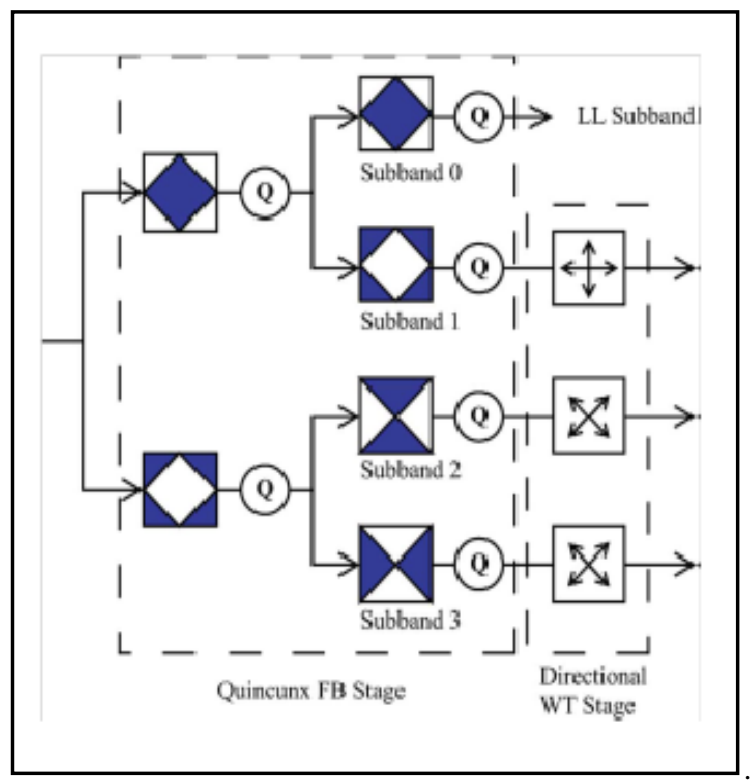

Fig3. Q-TODFB Decomposition.

\subsubsection{Directional 1-D Stage In D-TODFB}

Similar to the Q-TODFB, the directional wavelet transform stage is required to transform the high pass sub bands along the specific directions. We describe the transform directions for three high-frequency resolutions with taking into account downsampling matrices for diagonally quadrant FBs.

\subsubsection{Directions FOR LH Sub Band}

The frequency plane partitions for the LH region after the transform by the diagonally quadrant FBs. The main frequency directions are $(1,2)$ and $(-1,2)$; hence, the main curve directions are $(2,1)$ and $(-2,1)$, respectively. The horizontal downsampling corresponding to the filtering by the diagonally quadrant $\mathrm{FB}$ is applied to this sub band. After that, each sub band in LH halves the number of columns. In other words, if we have the curve direction before the horizontal downsampling, it becomes after it. Finally, in this case, the curve directions are changed into $(1,1)$ and $(1,1)$. Consequently, the two down- sampled LH resolutions are further transformed by the directional wavelet transform along the directions $(1,1)$ and $(-1,1)$. Consequently, the two down- sampled LH resolutions are further transformed by the directional wavelet transform along the directions $(1,1)$ and $(1$, $1)$, respectively,

\subsubsection{Directions For HL Sub Band}


Similar to the LH sub band, the curve directions after filtering by the diagonally quadrant FB are $(2,1)$ and $(-2,1)$, respectively. With the vertical down sampling $\operatorname{diag}(1,2)$, they are changed into $(1,1)$ and $(-1,1)$. Finally, these sub bands are transformed by the directional wavelet transform along the directions $(1,1)$ and $(1,-1)$ with horizontal downsampling. The entire downsampling matrix is also diag $(2,2)$.

\subsubsection{Directions for HH Sub Band}

In contrast to the other two high-frequency regions, the HH sub band requires different manipulations for the directional wavelet transform. Before down sampling for the diagonally quadrant $\mathrm{FB}$, the main frequency directions are $(1,1)$ and $(-1,1)$, and their curve directions are $(-1,1)$ and $(1,1)$, respectively. In this paper, the horizontal downsampling is considered. After that, the curve directions are changed into $(1,2)$ and $(1,2)$. The directional wavelet transform requires the vertical downsampling to yield the downsampling matrix diag $(2,2)$. Unfortunately, the directional wavelet transforms along $(-1,2)$ and $(1,2)$ with the vertical downsampling are not permitted since the $(-1,2)$ and $(1,2)$. Require even (odd) rows for the even (odd) row to be transformed.

\section{MULTI WAVELET TRANSFORM AND ITERATION OF DECOMPOSITION}

Dyadic wavelet decomposition is achieved by iterative two-channel perfect reconstruction filter bank operations over the low frequency band at each level. This leads to a full tree decomposition as depicted in Fig4. The final decomposition structure will be a subset of that full tree. Multi wavelets provide one alternative to the wavelet transform. Despite its general success, the wavelet transform often fails to accurately capture high frequency information, especially at low bit rates where such information is lost in quantization noise. A multilevel wavelet filter bank involves iterating the low pass-high pass filtering and down sampling.

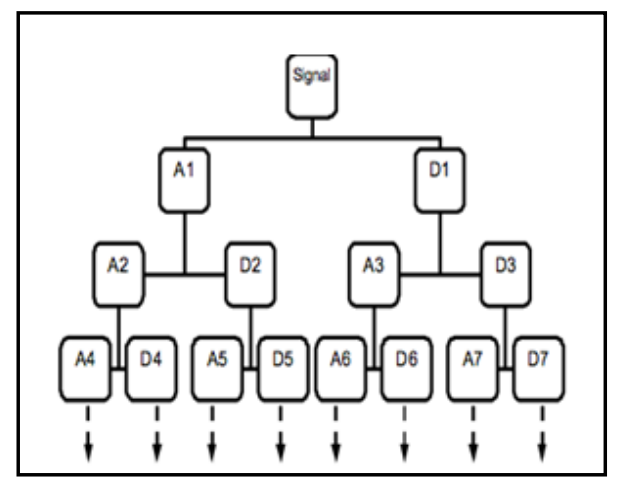

Fig4.Wavelet Packet Decomposition Tree

Since multi wavelet decomposition produce two low pass sub bands and two high pass sub bands in each dimension, the organization and statistics of multi wavelet sub bands differ from the scalar wavelet case.During a single level of decomposition using scalar wavelet transform, the 2-D image data is replaced with four blocks corresponding to the sub bands representing either low pass or high pass in both dimensions. These sub bands are illustrated in Figure 6-a.

The data in sub band LH 'was obtained from high pass filtering of the rows and then by low pass filtering of the columns. The multi wavelets used here have two channels, so there will be two sets of scaling coefficients and two sets of wavelet coefficients. The multi wavelet decomposition sub bands are shown in Figure 6-b.

For multi wavelets, the $\mathrm{L}$ and $\mathrm{H}$ labels have subscripts denoting the channel to which the data corresponds. For example, the sub band labeled $\mathrm{L} 1 \mathrm{H} 2$ corresponds to data from the second channel high pass filter in the horizontal direction and the first channel low pass in the vertical direction.

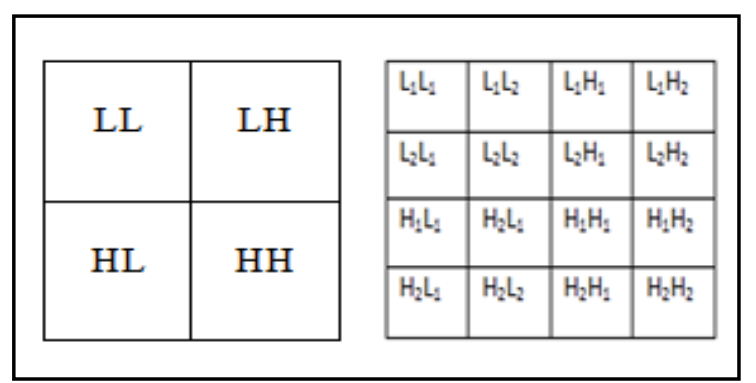

Fig5.ImageSub Bands after Single-Level Decomposition for (a) Scalar Wavelets (b) Multi Wavelets.

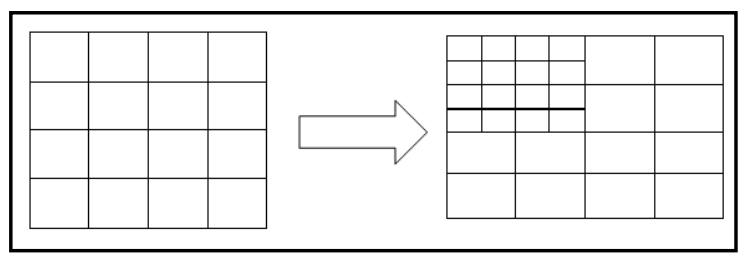

(a)

(b)

Fig6. Conventional iteration of multi wavelet decomposition

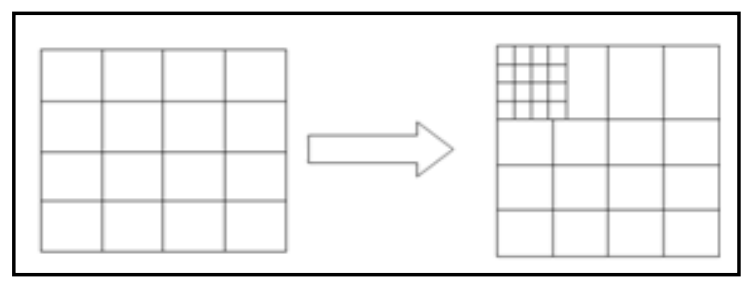

(a)

(b)

Fig7. Proposed iteration method for multiwavelet decomposition. Compare to fig6.

The multi wavelet decomposition sub bands are shown in Fig5. (b). For multi wavelets, the labels have subscripts denoting the channel to which the data corresponds. For example, the sub band labeled corresponds to data from the second channel high pass filter in the horizontal direction and the first channel low pass filter in the vertical direction. Scalar wavelet transforms give a single quarter-sized low pass sub band from the original larger sub band, as seen in sub band in Fig5.(a). The multiwavelet decompositions iterate on the low pass coefficients from the previous decomposition, [the sub bands in Fig.5 (b)]. In the case of scalar wavelets, the low pass quarter image is a single sub band. But when the multiwavelet transform is used, the quarter image of low pass coefficients is actually a block of sub bands one low pass and three band pass. The scaling function associated with the second channel is band pass since its anti symmetric form gives a zero. Two conclusions may be drawn from these observations. First, since these four sub bands possess different statistical characteristics, mixing them together using the standard multiwavelet decomposition results in subsequent sub bands with mixed data characteristics. This implies that typical quantization schemes that assume the statistics in each sub band are either low pass or high pass will not give the best possible results. Second, since only the sub band is entirely comprised of low pass characteristics, we only need to perform further iterations on that one sub band.

\section{PERFORMANCE MEASURES}

The phrase peak signal-to-noise ratio, often abbreviated PSNR, is an engineering term for the ratio between the maximum 
possible power of a signal and the power of corrupting noise that affects the fidelity of its representation. Because many signals have a very wide dynamic range, PSNR is usually expressed in terms of the logarithmic decibel scale.

The PSNR is most commonly used as a measure of quality of reconstruction of lossy compression codec's (e.g., for image compression). The signal in this case is the original data, and the noise is the error introduced by compression. When comparing compression codec's it is used as an approximation to human perception of reconstruction quality, therefore in some cases one reconstruction may appear to be closer to the original than another, even though it has a lower PSNR (a higher PSNR would normally indicate that the reconstruction is of higher quality). One has to be extremely careful with the range of validity of this metric; it is only conclusively valid when it is used to compare results from the same codec and same content. It is most easily defined via the mean squared error (MSE) which for two $m \times n$ monochrome images $\mathrm{I}$ and $\mathrm{K}$ where one of the images is considered a noisy approximation of the other is defined as

$$
M S E=\frac{1}{m n} \sum_{i=0}^{m-1} \sum_{j=0}^{n-1}[I(i, j)-K(i, j)]^{2}
$$

The PSNR is defined as:

$$
\begin{aligned}
P S N R & =10 \cdot \log _{10}\left(\frac{M A X_{I}^{2}}{M S E}\right) \\
& =20 \cdot \log _{10}\left(\frac{M A X_{I}}{\sqrt{M S E}}\right)
\end{aligned}
$$

Here, $M A X_{I}$ is the maximum possible pixel value of the image. When the pixels are represented using 8 bits per sample, this is 255. More generally, when samples are represented using linear PCM with $B$ bits per sample, $M A X_{I}$ is $2 \mathrm{~B}-1$. For color images with three RGB values per pixel, the definition of PSNR is the same except the MSE is the sum over all squared value differences divided by image size and by three. Typical values for the PSNR in lossy image and video compression are between 20 and $50 \mathrm{~dB}$, where higher is better. Acceptable values for wireless transmission quality loss are considered to be about 20 $\mathrm{dB}$ to $25 \mathrm{~dB}$. When the two images are identical the MSE will be zero for this value the PSNR is undefined.

\section{SIMULATION RESULTS}

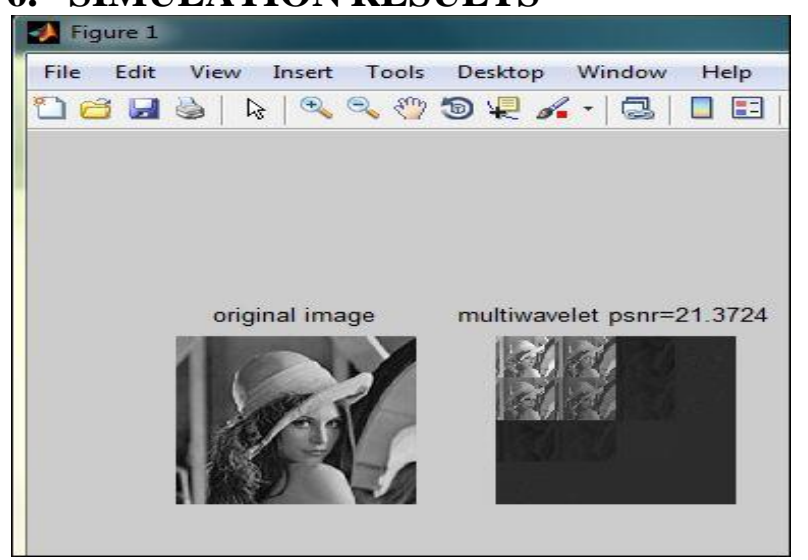

(a)

(b)

Fig8. (a) original image

(b) output image

Fig8. (a) shows the original image and the Fig8. (b) represents the output image in the low-low i.e. (LL) sub band. Hence the PSNR value of the output image is 21.38. The higher PSNR value of the image will have better visual results.
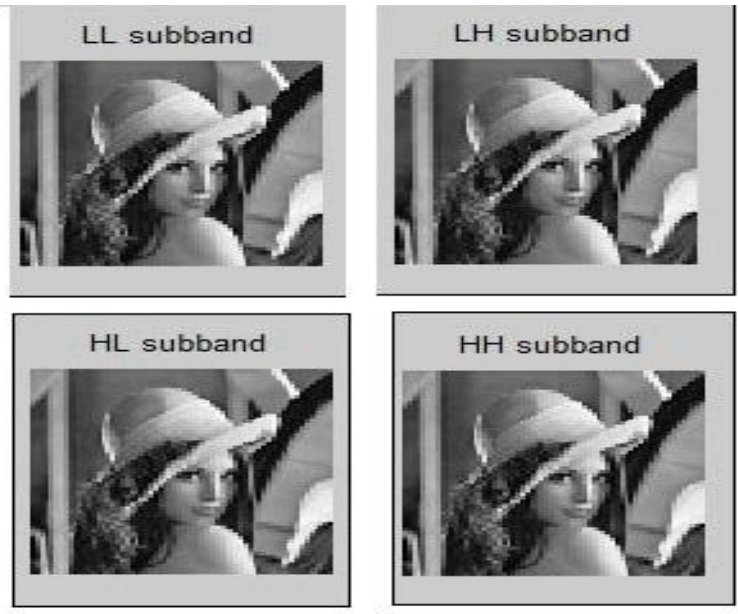

Fig9. Decomposition output of each sub band

Fig9. shows that the one-level decomposition of the original image. Each sub band is represented with a low pass and high pass combinations.

\section{COMPARISON OF RESULTS}

\begin{tabular}{|c|c|l|r|r|}
\hline \multirow{2}{*}{ Function } & \multicolumn{3}{|c|}{ Parameter } \\
\cline { 2 - 5 } & PSNR & $\begin{array}{c}\text { TIME } \\
(\mathrm{dec})\end{array}$ & $\begin{array}{c}\text { PSNR } \\
(\mathrm{db})\end{array}$ & $\begin{array}{c}\text { TIME } \\
(\mathrm{sec})\end{array}$ \\
\hline MULTI & 25.65 & 1.29 & 21.38 & 1.01 \\
WAVELET & & & & \\
\hline Q-TODFB & 31.39 & 0.88 & 42.76 & 1.35 \\
\hline D-TODFB & 34.83 & 0.85 & 35.12 & 1.14 \\
\hline
\end{tabular}

\section{TABLE I}

\section{CONCLUSION}

The performance of multi wavelets in general depends on the image characteristics. Where in the above compared result shows that the decrease in PSNR will be the same in time. By using this same lena image both the parameters are in high compared to existing. However multi wavelets appear to excel at preserving high frequency content. In particular, multi wavelets better capture the sharp edges and geometric patterns that occur in images. A new method for improving the multi wavelet transform have been proposed in this paper i.e., multi wavelet decomposition that iterates only on the sub band, Hence the proposed system is very efficient.

\section{REFERENCES}

[1] P. P. Vaidyanathan, Multirate Systems and Filter Banks. Englewood Cliffs, NJ: Prentice-Hall, 1993.

[2] G. Strang and T. Q. Nguyen,Wavelets and Filter Banks. Boston, MA: Wellesley-Cambridge, 1996.

[3] Directional multiresolution image representation," IEEE Trans. Image Process. vol. 14, no. 12, pp. 2091-2106, Dec. 
2005

[Online].

Available:http://www.ifp.uiuc.edu/ minhdo/software/

[4] Y. Lu and M. N. Do, "CRISP-contourlet: A critically sampled directional multiresolution image representation," in Proc. SPIE Conf. Wavelet Applications in Signal and Image Processing, 2003, pp. 655-665.

[5] T. T. Nguyen and S. Oraintara, "A class of multiresolution directional filter bank," IEEE Trans. Signal Process., vol. 55, no. 3, pp. 949-961, Mar. 2007.

[6] R. H. Bamberger and M. J. T. Smith, "A filter bank for the directional decomposition of images: Theory and design,"
IEEE Trans. Signal Process., vol. 40, no. 4, pp. 882-893, Apr. 1992.

[7] D. Wang, L. Zhang, A. Vincent, and F. Speranza, "Curved Wavelet transform for image coding," IEEE Trans. Image Process., vol. 15, no.8, pp. 2413-2421, Aug. 2006.

[8] W. Ding, F. Wu, and S. Li, "Lifting-based wavelet transform with directionally spatial prediction," in Proc. Picture Coding Symp., 2004, pp. 483-488.

[9] C.-L. Chang and B. Girod, "Direction-adaptive discrete wavelet transform for image compression," IEEE Trans. Image Process., vol. 16, no.5 , pp. 1289-1302, May 2007. 